\title{
3 Empirical Wave Propagation
}

The purpose of this chapter is to demonstrate that, given a long data set of global extent, one can design a simple forecast method called Empirical Wave Propagation (EWP), which has modest forecast skill and allows us to explore aspects of atmospheric dynamics empirically, most notably aspects that help to explain mechanisms of teleconnection. The highlight of this chapter are dispersion experiments where we ask the question what happens to an isolated source at $\mathrm{t}=0$. Even though nature has never done such an experiment, we will address this question empirically. In case the reader does not need/want to know the technical details of deriving wavespeeds he/she can skip to page 6 (EWP diagnostics) of this chapter... We will also discuss the skill of one-day EWP forecasts, in comparison to skill controls like 'persistence', as a function of season, hemisphere, level and variable. While short range forecasts are certainly not the topic of this book, we note that the short-term wave propagation features described here do nourish and maintain the teleconnection patterns thought to be important for longer range forecasts.

EWP uses either zonal harmonic waves ( $\sin / \cos$ pairs) along each latitude circle separately (EWP1), or global domain spherical harmonics (see Parkinson and Washington (1986) for basics on spherical harmonics), EWP2. The orthogonal functions used here are thus analytical. The atmosphere is to first order rotation-symmetric and obviously periodic in the east-west direction, which makes the zonal Fourier transform a natural. Moreover, many weather systems, wave-like in the upper levels, are seen to move from west to east (east to west) in the mid-latitudes (tropics), so a decomposition in $\sin / \cos$ functions should inform us about phase propagation and energy dispersion on the sphere. For any initial time we decompose the state of the atmosphere into harmonic waves. If we knew the wave speed, and make an assumption about the future amplitude, we could make forecasts by analytical means ${ }^{1}$. But how do we know the phase speed? One way to proceed, with data alone, is to calculate from a large data set the climatological

\footnotetext{
${ }^{1}$ There is an implicit assumption that waves of different wavenumber travel independently, i.e. no non-linear interaction.
}

\section{Ch. 3 Pg. 1}


speeds of anomaly ${ }^{2}$ waves. This is where the empirical aspects come in. Phase speed estimates can be made via a technique called phase shifting.

\subsection{Data and EWP Method}

\subsubsection{Data treatment.}

Consider a data set of, for example, $500 \mathrm{mb}$ height analyses (treated as 'observed'), once daily at $0 Z$, on a $2.5^{\circ} \times 2.5^{\circ}$ lat/lon grid, denoted as $Z(\lambda, \phi, t$, year), where $\lambda, \phi$ are longitude and latitude. Choosing just a small window in the annual cycle (+/- 15 days) around January 15, we can combine all January days during 1979-1995 into one single data set and have $t=1,31$. We now form anomalies by:

$$
\mathrm{Z}^{\prime}(\lambda, \phi, \mathrm{t}, \text { year })=\mathrm{Z}(\lambda, \phi, \mathrm{t}, \text { year })-\mathrm{Z}_{\text {climo }}(\lambda, \phi, \mathrm{t})
$$

where $Z_{\text {climo }}(\lambda, \phi, t)$ is based on a long multi-year data set $Z(\lambda, \phi, p$-level, day of the year, hour of the day .......). See Schemm et al(1997) for details on how such climatologies are prepared. Global Reanalysis (Kalnay et al 1996) and CDAS, its continuation in real time (Kistler et al 2001), allow us to choose any sub-period during 1948-present. We now select data along just one latitude circle at a time, a periodic domain: $Z^{\prime}(\lambda, 50 \mathrm{~N}, \mathrm{t}$, year). We further simplify notation to $Z^{\prime}(\lambda, t)$.

As in Eq (2.6) we project anomaly data Z' onto the sin / cos orthogonal pair for each $\mathrm{t}$. This yields two coefficients (a and b), or, alternatively, an amplitude (A) and a phase ( $\epsilon$ ) for each $\mathrm{m}, \mathrm{m}=0$ to 72 , i.e.

$Z^{\prime}(\lambda, t)=A_{0}(t)+\sum_{m} a_{m}(t) \cos m x+b_{m}(t) \sin m x=A_{0}(t)+\sum_{m} A_{m}(t) \cos m\left(x-\epsilon_{m}(t)\right)$

\footnotetext{
${ }^{2}$ Anomaly is defined as a departure from a climatological mean.
}

Ch. 3 Pg. 2 
where $\mathrm{x}=2 \pi \lambda / 360$, and $\lambda=0,2.5,5 \ldots \ldots$ 357.5. $\mathrm{A}_{0}$ is the zonal mean of $Z$, sometimes referred to as wavenumber $0 . \epsilon_{\mathrm{m}}$ is the phase angle. (3.1) is a classical Fourier transform or harmonic analysis of $Z^{\prime}(\lambda, t)$. Recall, $Z^{\prime}, A_{m}$ and $\epsilon_{m}$ are all functions of time.

\subsubsection{Amplitude.}

Essentially, in view of (3.1), in order to forecast Z'( $\lambda, t+1)$ given $Z^{\prime}(\lambda, t)$, we seek information about the amplitude $A_{m}$ and the phase $\epsilon_{m}$ at $t+1$. Splitting up the forecast problem explicitly into these two aspects $(A, \epsilon)$ is not all that common, but leads to special insights. The real forecast skill resides in the propagation aspect, while the skill related to the amplitude, in data studies, is usually just damping. Nevertheless we can learn from studying first the amplitude. In view of Parceval's theorem Eq (2.16), we can write the space time variance (STV) as:

$$
\begin{aligned}
& \mathrm{STV}=\underset{\mathrm{t} \mathrm{S}}{\Sigma \Sigma \mathrm{Z}^{, 2} /\left(\mathrm{n}_{\mathrm{s}} \mathrm{n}_{\mathrm{t}}\right)=} \underset{\mathrm{t} \mathrm{m}}{\frac{1}{2} \mathrm{~A}_{\mathrm{m}}^{2}} \\
& =1 / 2 \Sigma\left(<\mathrm{A}_{\mathrm{m}}>^{2}+<\mathrm{A}_{\mathrm{m}}{ }^{2}>\right), \\
& \mathrm{m}
\end{aligned}
$$

where $<>$ is the time mean, and $\mathrm{A}^{\prime}=\mathrm{A}-<\mathrm{A}>$.

From numerous calculations with many variables we find that about $75 \%$ or more of the variance in the atmosphere is associated with $<\mathrm{A}_{\mathrm{m}}>^{2}$, i.e., the observed variability can be thought of as anomaly waves with amplitude fixed at their climatological value, $<\mathrm{A}_{\mathrm{m}}>$, residing at some phase. (An example of this calculation is forthcoming in the discussion of Table 3.1). The remainder, $<\mathrm{A}_{\mathrm{m}}{ }_{\mathrm{m}}^{2}>$, due to amplitude variations is $25 \%$ or less, depending on variable. This certainly creates, by and large, the impression of stable waves, and therefore the prediction as one of primarily the phasing of waves. Striking 'development' localized in space (such as a suddenly growing cyclone) has to be mainly one of constructive interference, not one of periodic sin/cosine wave amplitude development. This point of view is in agreement with Farrell (1984) who was one of the first to question whether 'modal' (i.e. $\sin / \cos$ ) instability is the cause of mid-latitude cyclone

\section{Ch. 3 Pg. 3}


development, a view that had been held since the 1940s.

Please note the $2^{\text {nd }}$ layer of climatology in $\mathrm{Eq}$ (3.2). We had already removed $\mathrm{Z}_{\text {climo }}$ from $\mathrm{Z}$, then defined the climatology of the amplitude of anomaly waves (a $2^{\text {nd }}$ moment). In contrast to $<\mathrm{a}_{\mathrm{m}}>$ and $\left\langle\mathrm{b}_{\mathrm{m}}>,<\mathrm{A}_{\mathrm{m}}>\right.$ is not zero because amplitude is derived from a squared quantity (i.e. note that Eq (3.2) does not refer to the amplitude of climatological mean waves in Z.)

Now, take a single anomaly wave $A_{m} \cos m\left(x-\epsilon_{m}\right)$. The question: will this wave move east or west and by how much per unit time. The question cannot be trivially answered by studying data because a) the speed varies greatly from day-to-day, and b) ambiguities arise when the wave moves more than $180^{\circ}$ to the east (or west), relative to its own wavelength. To lessen these problems we use a 'phase shifting' technique.

\subsubsection{Phase shifting}

Following Van den Dool and Qin(1996), and dropping the m index in A and $\epsilon$ for simplicity, we consider a single wave $\mathrm{m}$ and write:

At time t: $\quad A \cos m(x-\epsilon)=a \cos m x+b \sin m x$

At time $\mathrm{t}+1: \quad \mathrm{A}^{+1} \cos \mathrm{m}\left(\mathrm{x}-\epsilon^{+1}\right)=\mathrm{a}^{+1} \cos \mathrm{mx}+\mathrm{b}^{+1} \sin \mathrm{mx}$

Now move the crest of the wave at time $t$ to a reference longitude (Greenwich for instance) - this is done by phase shifting over $+\epsilon$. Move the wave on the next time level $(\mathrm{t}+1)$ over the same angle $\epsilon$ - this maintains the relative positioning of the waves at successive days, but in a new framework. Phase shifting yields:

At time t: $\quad A \cos m(x)=A \cos m x+0 \sin m x$

At time $\mathrm{t}+1: \mathrm{A}^{+1} \cos \mathrm{m}\left(\mathrm{x}-\left(\epsilon^{+1}-\epsilon\right)\right)=\mathrm{c}^{+1} \cos \mathrm{mx}+\mathrm{d}^{+1} \sin \mathrm{mx}$

where $\mathrm{c}^{+1}=\mathrm{a}^{+1} \cos \mathrm{m} \epsilon+\mathrm{b}^{+1} \sin \mathrm{m} \epsilon$ and $\mathrm{d}^{+1}=\mathrm{b}^{+1} \cos \mathrm{m} \epsilon-\mathrm{a}^{+1} \sin \mathrm{m} \epsilon$.

The phase shifting is done for all pairs $t / t+1$ (all 510 pairs for say January 1979-1995 for instance) and $\epsilon$ is always the phase angle on the leading day. The r.h.s. coefficients in (3.4) and (3.4a), A, $\mathrm{c}^{+1}$ and $\mathrm{d}^{+1}$, are a function of time, with time means $\langle\mathrm{A}\rangle,\left\langle\mathrm{c}^{+1}\right\rangle$ and $\left\langle\mathrm{d}^{+1}\right\rangle$. The time

\section{Ch. 3 Pg. 4}


mean of coefficients $a$ and $b\left(\right.$ and $\mathrm{a}^{+1}$ and $\mathrm{b}^{+1}$ ) would be very nearly zero. All variables we have introduced in (3.3) and (3.4) can be evaluated from the data.

Amplitudes of the time averaged phase shifted (subscript ps) wave $m$ are given by:

$$
\begin{array}{ll}
\mathrm{t}: & \mathrm{A}_{\mathrm{ps}}=<\mathrm{A}> \\
\mathrm{t}+1: & \mathrm{A}_{\mathrm{ps}}^{+1}=\sqrt{\left(<\mathrm{c}^{+1}>^{2}+<\mathrm{d}^{+1}>^{2}\right)}
\end{array}
$$

Phase angles of the time averaged phase shifted wave are given by:

$$
\begin{array}{ll}
\mathrm{t}: & \epsilon_{\mathrm{ps}}=0 \\
\mathrm{t}+1 & \epsilon_{\mathrm{ps}}^{+1}=\arctan \left(<\mathrm{d}^{+1}>/<\mathrm{c}^{+1}>\right)
\end{array}
$$

The resulting $\mathrm{A}_{\mathrm{ps}}$ and $\epsilon_{\mathrm{ps}}$ can be generated for each $\mathrm{m}$. The amplitude at time $\mathrm{t}$ is not changed by the phase shifting: $A_{p s}$ is the same as $<A_{m}>$ in (3.2).

If the wave at $\mathrm{t}+1$ were in a random phase relative to the wave at $\mathrm{t},\left\langle\mathrm{c}^{+1}\right\rangle$ and $\left\langle\mathrm{d}^{+1}\right\rangle$ would be zero and hence $\mathrm{A}_{\mathrm{ps}}{ }^{+1}$ would be zero. The ratio $\mathrm{A}_{\mathrm{ps}}{ }^{+1} / \mathrm{A}_{\mathrm{ps}}$ thus tells us the degree of nonrandomness in $\left(\epsilon^{+1}-\epsilon\right)$ or the steadiness in propagation for the given time increment.

The phase shifting technique is helpful mainly because it postpones dealing with the ambiguity about displacement larger than $+/-180^{\circ}$, until after the time averaging of $\mathrm{c}^{+1}$ and $\mathrm{d}^{+1}$. On many individual days with either high phase speed and/or low amplitude waves (or too large time increment) the ambiguity is difficult to deal with.

EWP is related to time spectral analysis but uses only short-time increment lagged data to determine wave speeds under quasi linear conditions.

\subsubsection{Mean propagation}

The phase speed $\mathbb{C}$ (in $\mathrm{m} / \mathrm{s}$ ) can be obtained from $\epsilon_{\mathrm{p}}^{+1}$ (in (3.5d) in radians) as

$$
\mathbb{C}(\phi, \mathrm{m})=\epsilon_{\mathrm{ps}}{ }^{+1}(\mathrm{~m}, \phi) .6375000 \cdot \cos (\phi) / 86400 / \mathrm{m}
$$

where the constants are the radius of the earth and number of seconds per day (since we used data once daily). When using spherical harmonics instead of $\sin / \mathrm{cos}$ only the speed at the equator needs to be reported. In all cases $\mathrm{A}_{\mathrm{ps}}{ }^{+1}<\mathrm{A}_{\mathrm{ps}}$, i.e using this method, anomaly wave amplitudes are

\section{Ch. 3 Pg. 5}


always damped. Some degree of damping is typical for statistical methods. All waves appear stable. Damping is small (large) for long(short) waves. We have found that a 1-day time increment works very well. For larger time spacing (2 days....10 days), the damping increases quickly.

\subsubsection{EWP forecast method.}

To apply EWP as a forecast method it is enough to know $\epsilon_{\mathrm{ps}}{ }^{+1}$ as a function of wavenumber and latitude for a given time of year. I.e on independent data we decompose the anomaly height field at $\mathrm{t}=0$ into waves, using (2.6), then move each wave by $\epsilon_{\mathrm{ps}}{ }^{+1}(\mathrm{~m}, \phi)$ from $(3.5 \mathrm{~d})$, then use (3.1) to arrive at a forecast in physical space for $\mathrm{t}=1$. The very same forecast method could also be applied if $\epsilon_{\mathrm{ps}}{ }^{+1}(\mathrm{~m}, \phi)$ were known from theory, as is the case for a simple model (barotropic) in a simple basic state (like $\mathrm{U}(\phi)=\mathrm{U}_{\mathrm{eq}} \cos \phi$, a state called super rotation). EWP is an analytical prediction method, and the word empirical applies only to the source of information that yields $\epsilon_{\mathrm{ps}}^{+1}$.

In the above we presented and derived the EWP forecast method along intuitive lines. In Appendix 3.1, we also present a formal derivation based on rmse minimization with very nearly the same result.

\subsection{EWP diagnostics.}

Table 3.1 serves as an example of the diagnostic aspects of EWP. We analyzed 20 years of 500mb data for January $1968-1987$. Information is given here for $50^{\circ} \mathrm{N}$, for selected zonal wavenumbers $\mathrm{m}=0,1,3,5,7,9,11$. For quick comprehension all numbers are rounded off to the nearest integer.

Table 3.1 Tabulation of amplitude, \% variance, phase angle propagation, phase speed and amplitude ratio, for selected zonal wavenumbers of daily $500 \mathrm{mb}$ height anomalies in January for $50^{\circ} \mathrm{N}$. The time increment is 24 hours. Period $=1969-1987 . \mathrm{ND}=$ Not Defined.

\begin{tabular}{lccccccccc}
$1 \mathrm{~m}->$ & 0 & 1 & 3 & 5 & 7 & 9 & 11 & units & Reference \\
\hline$A_{\mathrm{m}}>$ or $A_{p s}$ & 26 & 73 & 73 & 57 & 35 & 21 & 13 & $\mathrm{gpm}$ & $(3.5 \mathrm{a})$
\end{tabular}

Ch. 3 Pg. 6 


\begin{tabular}{|c|c|c|c|c|c|c|c|c|c|}
\hline$<A_{\mathrm{m}}>^{2} /<$ & 63 & 80 & 79 & 82 & 80 & 77 & 77 & $\%$ & $(3.2)$ \\
\hline$\epsilon_{p s}^{+1}$ & $N D$ & -3 & 3 & 31 & 71 & 108 & 136 & ${ }^{o} \lambda$ & $(3.5 d)$ \\
\hline $\mathbb{C}$ & $N D$ & -3 & 1 & 5 & 8 & 10 & 10 & $\mathrm{~m} / \mathrm{s}$ & (3.6) \\
\hline$A_{p s}^{+1} / A_{p s}$ & 89 & 90 & 88 & 79 & 65 & 44 & 32 & $\%$ & $(3.5 a / b)$ \\
\hline
\end{tabular}

The time mean amplitude $<\mathrm{A}_{\mathrm{m}}>$ is given in the first line, and the fraction of variance represented by $<\mathrm{A}_{\mathrm{m}}>$ in the second line. The long waves have large amplitude, nearly constant for $\mathrm{m}=1$ to 4 at 70-75 geopotential meter (gpm) while amplitude drops off sharply with $\mathrm{m}$ beyond wavenumber 4. As shown in the $2^{\text {nd }}$ line the time mean amplitude of the anomaly waves represents $75-80 \%$ of the variance (except in the zonal mean $(\mathrm{m}=0)$ where the percentage is only $63 \%$ ). The phase propagation, in degrees relative to own wavelength denoted ${ }^{\circ} \lambda$, is given in the $3^{\text {rd }}$ line and the conversion to speed in $\mathrm{m} / \mathrm{s}$ in the $4^{\text {th }}$ line. Long waves travel westward (-), and short waves eastward $(+)$, in good qualitative agreement with the theoretical Rossby equation for mid-latitudes (Holton 1979 p167), which reads $\mathbb{C}=U-\beta / K^{2}$, where $\mathbb{C}$ is the phase speed, $U$ is the background windspeed, $\beta$ is the meridional derivative of the Coriolis parameter, and $\mathrm{K}$ is wavenumber (if only the zonal wavenumber is considered $K$ relates to $\mathrm{m}$ as $\mathrm{K}=2 \pi \mathrm{m} / \mathrm{L}$, where $\mathrm{L}$ is the length of the latitude circle.) The short wave speed (large $\mathrm{K}$ or $\mathrm{m}$ ) is nearly constant with $\mathrm{m}$ at $10 \mathrm{~m} / \mathrm{s}$ and no ambiguities arise for the wavenumbers shown, the largest displacement shown being $136^{\circ}$ or less than half the wavelength, even for $m=11$. The displacement in degrees depends obviously on the time increment $(\Delta \mathrm{t})$, chosen here as one day, but the speed $\mathbb{C}$ in $\mathrm{m} / \mathrm{s}$ depends barely on $\Delta \mathrm{t}$ as long as $\Delta \mathrm{t}$ is small. As can be judged from the $5^{\text {th }}$ line: The phase propagation is rather steady (large $\mathrm{A}_{\mathrm{p}}^{+1} / \mathrm{A}_{\mathrm{p}}$ ) for the long waves, but is increasingly more variable and harder to determine for the short waves.

The fact that wave speed depends on $\mathrm{m}$, or the wavelength, is called dispersion and leads to most interesting consequences described later on.

$<$ deleted text $>$

Next we report on using spherical harmonics. For these functions, $\sin / \cos$ in longitude Ch. 3 Pg. 7 
and associated Legendre functions in latitude, we have two wavenumbers to consider. Therefore in addition to $\mathrm{m}$ there is also the (total) wavenumber $\mathrm{n}(\mathrm{n}-\mathrm{m}$ is the number of zero crossings between the two poles). On the other hand, results are simpler in that they apply to the whole sphere at once and there is thus no need to discuss $50^{\circ} \mathrm{N}, 50^{\circ} \mathrm{S}$ and Equator separately. Table 3.5 shows zonal (west to east) phase speeds when using spherical harmonics.

Table 3.5 Tabulation of phase speed $\mathbb{C}$ for selected spherical harmonics of global daily 500mb height anomalies in January. The time increment is 24 hours. Period $=1969-1987 . N D=N o t$ Defined. Units are $\mathrm{m} / \mathrm{s}$, and the reference is Eq 3.6. The speeds shown are valid at the equatorspeeds at other latitudes are obtained by multiplication by cos $\phi$.

$\begin{array}{lllllll}\mid m-> & 1 & 3 & 5 & 7 & 9 & 11 \\ n=1 & -35 & N D & N D & N D & N D & N D \\ n=3 & -13 & -9 & N D & N D & N D & N D \\ n=5 & -4 & -3 & 0 & N D & N D & N D \\ n=7 & 2 & 3 & 5 & 4 & N D & N D \\ n=9 & 3 & 6 & 9 & 9 & 3 & N D \\ n=11 & 6 & 9 & 11 & 13 & 9 & 3 \\ n=13 & 7 & 9 & 11 & 16 & 14 & 9 \\ n=15 & 9 & 9 & 13 & 16 & 18 & 13\end{array}$

Clearly spherical harmonics obtain an even better separation in westward moving long waves and eastward moving short waves. For instance, depending on $n$, zonal wavenumber $m=1$ has speeds ranging from $-35 \mathrm{~m} / \mathrm{s}$ to $+7 \mathrm{~m} / \mathrm{s}$. Shorter zonal waves $(\mathrm{m}=9)$, if associated with short scales in the meridional direction as well, can reach phase speeds of $18 \mathrm{~m} / \mathrm{s}$ in January. While meridional phase speed is not defined for either zonal or spherical harmonics, the dependence of the zonal phase speed on $\mathrm{n}$ (as opposed to only $\mathrm{m}$ ) makes a major difference for wave propagation on a sphere. Theoretically (Baer 1972) the phase speed of spherical harmonics in a simple background flow depends on $n$ only, see inset 2 , but we find empirically a strong dependence on $\mathrm{m}$ as well.

\subsection{Rock in the pond experiments.}

Ch. 3 Pg. 8 
We are now ready for an experiment. A round disturbance is placed at $45^{\circ} \mathrm{N}$ on a polar stereographic map of the Northern Hemisphere, see Fig.3.1 upper left. (The values of the disturbance decrease from the maximum (150 in arbitrary units) as $\exp \left(-\sqrt{ } \mathrm{r} / \mathrm{r}_{0}\right)$, where $\mathrm{r}$ is distance to the center in degrees and $r_{0}$ is the e-folding radius, $r_{0}=7.5$ degrees. The center value (in arbitrary units) is 150 . The 20 contour extends about 15 degrees from the center.) One can think of this as an isolated anomaly $Z^{\prime}$ in $500 \mathrm{mb}$ height in January. The rest of the world has nearzero anomalies initially. The continental outlines are for orientation only - the experiment is zonally invariant. Contours are every 20 , no zero line shown. Units (and sign) are arbitrary because the method is linear. The question is what will happen to this initial source. If this were a passive tracer one might expect the blob to move along with the background wind. Dispersion by gravity waves would take the pressure perturbation in all directions. Here we will witness very different behavior. Decomposing the disturbance into twenty zonal waves, one can use EWP to propagate each wave at each latitude (at 2.5 degree spacing) by its own phase speed (using the complete version of Tables 3.1-3.3, all latitudes, all $\mathrm{m}$ ) while leaving the wave amplitude unchanged, and recompose the field one day later. After one day the original disturbance has moved east, but one may notice a downstream development of opposite sign and an upstream development of like sign. At day one we have, in a sense, three rocks in the pond, each of which is repeating the process. The downstream anomaly gains amplitude by day 2 and kicks off an anomaly further downstream. One can follow the peak of the original rock moving east until day 4. A wavetrain $(+,-,+)$ or traveling stormtrack plus envelope is seen at day 3 and beyond. Remarkably, the dispersion of stable waves leads to downstream development and even formation of strong gradients (frontogenesis). The upstream development of the same sign causes much persistence in the area of origin. Much of the variability (75-80\% of the variance) in the atmosphere can be 'explained' this way (stable waves moving around). Even though in nature one will never observe this experiment we have, by applying empiricism, found reasonable behavior and are able to demonstrate a number of physical processes. The dispersion causes the non-trivial

\section{Ch. 3 Pg. 9}


motion of the original anomaly. For the notion 'energy' think of $Z$ '2. This quantity is conserved in a space integrated sense, but one can see the energy travel at speeds higher than the phase speed through the wavetrain. This phenomenon is also called group velocity, see Holton(1979) p151. Comparison to numerical experiments by Simmons and Hoskins (1979) and Chang and Orlanski(1994) can be made. The shape and orientation of the eddies in the wavetrain is such that they would transport momentum (u'v') into the jet, so remarkably a linear empirical experiment shows features of non-linearity, similar to Branstator's (1995) eddy feedback model.

Fig.3.2 is the same experiment, but now the dispersion is two dimensional by using spherical harmonics instead of (as done in Fig.3.1) zonal harmonics by latitude. From the beginning the dispersion is different in character than in Fig.3.1. While zonal dispersion can still be seen, this 2D version of EWP also shows meridional energy propagation, i.e. the anomalies travel outside the latitude band in which they were contained at $\mathrm{t}=0$, and after a few days patterns emerge that look like veritable large scale teleconnections. This happens even though the phase speed is always in the zonal direction. After many days the NH source even kicks off wavetrains in the SH midlatitudes (not shown). Dependence of phase speed on $\mathrm{n}$ thus makes a very large difference for energy propagation. (For later reference: the energy travels in a direction perpendicular to the long axis of the anomaly ellipse.) Studies of teleconnections by Rossby wave propagation on the sphere in idealized numerical models were made by Opsteegh and Van den Dool(1980) and Hoskins and Karoly(1981). Many of their results could have been obtained with the even simpler EWP approach.

In both Fig.3.1 and 3.2 one may think of the experiment in the following way. By constructive and destructive interference, a set of global functions is made to be non-zero in a local area, and zero elsewhere. As soon as the clock starts ticking, the waves move and the degree of interference changes gradually. Because of dispersion the initial blob is not just translated (to the east or west) as a single entity but shows remarkable transformation, zonally as well as meridonally. 
We have essentially created a virtual laboratory experiment. The reader could place one or more sources wherever she/he wants to study propagation for a certain season and variable and then study what happens.

\subsection{Skill of EWP 1-day forecasts.}

Instead of using idealized initial states we can start from an observed anomaly field and make a 24 hour forecast which can be verified. We now discuss the skill of such EWP forecasts. The above discussion about wave energy dispersion and the basic processes of teleconnections would be enhanced if we can show that EWP has a certain forecast capability. Because of superior performance by Numerical Weather Prediction, in 2005, EWP is not a forecast tool of practical interest. Our control to judge skill is persistence (PER), i.e. persist yesterday's anomalies for 24 hours as a 'lazy man's forecast'. Both EWP and PER are verified over a very large number of cases using the anomaly correlation defined in chapter 2.

Table 3.6: Anomaly Correlation of 24 hr forecasts by EWP and Persistence (PER) for three domains, Northern and Southern Hemisphere (NH, SH) and Tropics (TR), as a function of variable and level. Data is for 0Z, 1979-1995 in December through February. Where the gain of EWP over PER exceeds 10 points the values are underlined bold.

\begin{tabular}{|c|c|c|c|c|c|c|c|c|c|}
\hline & \multicolumn{2}{|c|}{ Streamfunction } & \multicolumn{2}{|c|}{ Velocity Potential } & \multicolumn{2}{|c|}{ Geop Height } & \multicolumn{2}{|c|}{ Temperature } \\
\hline & & EWP & PER & EWP & PER & EWP & PER & EWP & PER \\
\hline \multirow{3}{*}{$50 \mathrm{mb}$} & $\mathrm{NH}$ & 95 & 94 & 64 & 64 & 94 & 93 & 92 & 91 \\
\hline & TR & 90 & 88 & 63 & 62 & 81 & 80 & 76 & 76 \\
\hline & $\mathrm{SH}$ & 94 & 93 & 52 & 51 & 91 & 89 & 85 & 79 \\
\hline & $\mathrm{NH}$ & 86 & 80 & 75 & 67 & 86 & 81 & 74 & 63 \\
\hline \multirow[t]{3}{*}{$200 \mathrm{mb}$} & TR & 86 & 84 & 80 & 78 & 86 & 83 & $\overline{73}$ & 68 \\
\hline & $\mathrm{SH}$ & $\underline{86}$ & 75 & 73 & 67 & 85 & 72 & $\underline{74}$ & 53 \\
\hline & $\mathrm{NH}$ & 84 & 78 & 55 & 52 & 83 & 77 & $\underline{71}$ & 60 \\
\hline
\end{tabular}

Ch. 3 Pg. 11 


\begin{tabular}{|c|c|c|c|c|c|c|c|c|}
\hline \multirow{2}{*}{$\begin{array}{r}500 \mathrm{mb} \mathrm{TR} \\
\mathrm{SH}\end{array}$} & 82 & 79 & 65 & 64 & 82 & 79 & 72 & 69 \\
\hline & 84 & 70 & 55 & 54 & 83 & 69 & 74 & 53 \\
\hline $\mathrm{NH}$ & 81 & 75 & 64 & 55 & 79 & 74 & 73 & 66 \\
\hline $850 \mathrm{mb}$ TR & 77 & 75 & 75 & 73 & 78 & 77 & 74 & 73 \\
\hline $\mathrm{SH}$ & $\underline{80}$ & 70 & 65 & 58 & 78 & 67 & 71 & 53 \\
\hline & Prec. & jater & vert $n$ & otion $500 \mathrm{mb}$ & surf p & $\begin{array}{l}---- \\
\text { essure }\end{array}$ & precip & tation \\
\hline $\mathrm{NH}$ & 50 & 32 & 16 & 6 & 76 & 70 & 15 & 5 \\
\hline $\mathrm{TR}$ & 76 & 73 & 41 & 40 & 78 & 75 & 41 & 40 \\
\hline $\mathrm{SH}$ & 55 & 31 & 18 & 6 & 75 & 63 & 18 & 6 \\
\hline & EWP & PER & EWP & PER & EWP & PER & EWP & PER \\
\hline
\end{tabular}

For all variables and levels EWP is better than PER. This is true even for every single forecast. Indeed EWP is a very safe and conservative forecast (wave dispersion is always in effect). The gains over PER are largest in the troposphere, the mid-latitudes, the southern hemisphere and for temperature. The largest gains are about 20 points. In the tropics, in the stratosphere, and for velocity potential EWP has very little skill over PER at day 1. At the bottom of Table 3.6 some assorted variables are listed. The surface pressure behaves consistent with heights aloft. In fact, phase speeds are nearly constant from sea-level to $50 \mathrm{mb}$ (not shown) as systems appear to travel with strong vertical coherence. Vertical motion and rainfall are nearly impossible to forecast, but even here some propagation can be surmised. Precipitable water in the atmosphere is not easy to forecast either, but EWP does have a large gain over PER.

We conclude the EWP describes realistic processes because it results in forecasts with a substantial gain in skill over persistence. If the reader feels that EWP is like a barotropic model, please note that EWP works equally well for many variables at many levels, not just Z500 in midlatitudes.

Fig.3.3 shows reduction in root-mean-square error (rmse) of EWP relative to PER as a function of wavenumber for the $\mathrm{NH}$ and $\mathrm{SH}$ along $50^{\circ}$. It is pretty obvious that the gains are due to mobile waves $m=4-13$. Without taking wave motion into account (PER) the error is large, while EWP accomodates the motion (if only in an averaged sense), cutting the rmse by up to 
$50 \%$. In the long waves EWP does not beat PER, even though the phase speed is non-zero. This is because it takes a high speed for a long wave to travel an appreciable distance relative to its own wavelength (which is what is needed to beat PER). Such speeds are not observed. The $+10 \mathrm{~m} / \mathrm{s}$ for short waves is worth a lot more in terms of forecast skill than the $-25 \mathrm{~m} / \mathrm{s}$ for the longest waves. Fig.3.3 is for EWP using zonal waves. Use of spherical harmonics lowers forecast skill everywhere! Apparently there is some merit in localizing the phase speed estimates.

\subsection{Discussion of EWP}

$<$ deleted text $>$

\subsubsection{Application of EWP}

It would be a stretch to believe that EWP has a wide practical application in forecasting. Its use is mainly for teaching and demonstration purposes. Nevertheless there are a few application with practical meaning based on EWP, which we list here.

a) NWP models have been weak at some specific features, such as the Madden and Julian Oscillation (MJO; Madden and Julian 1971), a global phenomenon in the tropics traveling eastward along the equator (Waliser et al 2005). Real time forecasts of the MJO by several methods including EWP can be found on the web. In the case of MJO, EWP was applied to $200 \mathrm{mb}$ velocity potential fields, see link at (http://ww.cpc.ncep.noaa.gov/products/precip/CWlink/MJO/mjo.html), and the article under readme. In spite of the strong westward speed reported for the height field (or streamfunction) in the tropics, see Table 3.3, the longest waves in velocity potential anomalies travel eastward with a speed that varies semi-annually between $5.5 \mathrm{~m} / \mathrm{s}$ (February and September) and $11 \mathrm{~m} / \mathrm{s}$ (May and November). These eastward speeds may indicate the dominance of Kelvin waves. Fig. 3.4 gives the near equatorial phase speeds of $200 \mathrm{mb}$ velocity potential anomalies for waves 1 - 30 in April. Long waves move eastwards, but short waves move westwards. The longest waves have the largest amplitude, and the amplitude is highest near the equator.

Ch. 3 Pg. 13 
b) Interpolation of weather maps provided only every 6 hours. EWP is much more accurate at interpolation than linear interpolation, see Van den Dool and Qin(1996). This EWP application will exist as long as model forecast and analysis results are provided infrequently ( every 6 hours). For potent small scale moving weather systems EWP is a good interpolation method even if weather maps were available every hour. EWP can likewise be applied to provide time interpolated boundary conditions for a limited area model, given global forecasts every 6 hours. c) In many geophysical disciplines there is a need to know atmospheric tides at high temporal resolution. Interpolation of very fast atmospheric tides (one revolution per day) from 6 hourly data to once hourly was addressed in Van den Dool et al(1997) and the results are applied in several geodetic and oceanographic research areas. Salvaging data originally sampled at the Nyquist frequency (the semi-diurnal tide dominates!) is a peculiar challenge that can be addressed using EWP.

d) the guess field for data assimilation. In data assimilation an NWP model is used to make the guess field. This is a drawback in case the model keeps introducing certain systematic errors. EWP could be used to advance the previous analysis for 3 or 6 hours, i.e. EWP could make the guess field.

\subsubsection{Weak points of EWP}

We hope the reader feels encouraged to interrogate a data set to come up with empirically based methods that can be used to explain difficult concepts, and to some degree to make forecasts. Certainly, EWP is not perfect. In listing weak points we hope some readers will be inspired to try to outdo something as deceptively simple as EWP.

1. EWP derives propagation properties without regard for longitude, i.e. for Fig. 3.1 it makes no difference where the source is situated relative to longitude or the standing waves (implicitly the

Ch. 3 Pg. 14 
land-ocean distribution).

2. Propagation speeds are independent of time (except the annual cycle). In view of the Rossby equation one might want to study time variation on the interannual time scale. In years with stronger jets, waves should move faster.

3. The meridional scale was neglected in EWP1 (zonal harmonics), and when it was included (EWP2, spherical harmonics) the forecast skill decreased. This appears to be because spherical harmonics give estimates as a compromise between two hemispheres (in different seasons), tropics and mid-latitude. An approach in a restricted latitude bands, like $20 \mathrm{~N}$ to the pole, with sine waves in the north-south direction (semi Fourier) would improve upon EWP1.

At a more fundamental level and beyond repair is the drawback that EWP does not deal with energy lost to or gained from the basic state. As an arching route is followed by a group of disturbances new energy added to the disturbance may be as large as the energy from the initial source. In Chapter 7 we will see that an empirical method called constructed analogue makes forecasts a la EWP, but some interaction with the basic state can be seen.

\section{Inset or Appendix $1:$ EWP formal derivation}

One can derive EWP formally by asking which constant propagation angle $\epsilon$ and damping factor $\rho$ should be applied to a wave at $\mathrm{t}=0$ so as to yield, on average over many cases, the best forecast of that wave at a future time. Here, best forecast means lowest rms error on average. The observed (phase shifted) waves at $\mathrm{t}=0$ and $\mathrm{t}=1$ being A $\cos \mathrm{x}$ and $\mathrm{c} \cos \mathrm{x}+\mathrm{d} \sin \mathrm{x}$, respectively, and the forecast for $\mathrm{t}=1$ based on observations at $\mathrm{t}=0$ being $\rho \mathrm{A} \cos (\mathrm{x}-\epsilon)$, one thus needs to minimize

$$
\mathrm{U}=\Sigma(\rho \mathrm{A} \cos \epsilon-\mathrm{c})^{2}+(\rho \mathrm{Asin} \epsilon-\mathrm{d})^{2}
$$

Ch. 3 Pg. 15 
In (3.a1) summation is over all times, $\mathrm{A}, \mathrm{c}, \mathrm{d}$ are a function of time (with non-zero means). $\epsilon$ and $\rho$ are constant in time. Upon differentiation wrt $\rho$ and $\epsilon$ one obtains:

$$
\Sigma \rho \mathrm{A}^{2}-\mathrm{c} A \cos \epsilon-\mathrm{d} \mathrm{A} \sin \epsilon=0
$$

and

$$
\Sigma \mathrm{c} A \sin \epsilon-\mathrm{d} A \cos \epsilon=0
$$

The solution (to be evaluated from a data set) is:

$$
\epsilon=\operatorname{atan}\{<\mathrm{dA}>/<\mathrm{cA}>\}
$$

where $<>$ are time means. Given $\epsilon$, the damping $\rho$ can be calculated as

$$
\rho=[<\mathrm{cA}>\cos \epsilon+<\mathrm{dA}>\sin \epsilon] /<\mathrm{A}^{2}>
$$

(3.a2) can also be written as

$$
\epsilon=\operatorname{atan}\left[\left\{<\mathrm{d}><\mathrm{A}>+<\mathrm{d}^{\prime} \mathrm{A}^{\prime}>\right\} /\left\{<\mathrm{c}><\mathrm{A}>+<\mathrm{c}^{\prime} \mathrm{A}^{\prime}>\right\}\right]
$$

If we neglect the covariance between $\mathrm{A}$ and both $\mathrm{c}$ and $\mathrm{d}$, the requirement for minimal $\mathrm{U}$ is $\epsilon=$ atan $\left\{<\mathrm{d}>/<\mathrm{c}>\right.$ ), which is EWP as proposed intuitively. Even if the transients terms like $<\mathrm{d}^{\prime} \mathrm{A}^{\prime}>$ are not small the resulting $\epsilon$ may not necessarily differ much from $\operatorname{atan}(<\mathrm{d}>/<\mathrm{c}>)$. An evaluation on the data used in Table 3.1-3.3 shows extremely minor influence from the covariances on the resulting $\epsilon$. The impact would be larger if, for instance, above average $A$ is associated with faster than average propagation - apparently this does not happen.

Note that damping the wave, $\rho<1$, for the purpose of lowering rms error does not change

Ch. 3 Pg. 16 
the requirements for optimal propagation. The damping, given by (3.a3), goes to zero for large time increments (say 10 days).

Two final comments: 1 . One can derive EWP formally with all the result shown above without phase shifting! The expressions are a little longer but otherwise the same. So while phase shifting is helpful, it is not necessary. 2. The rms minimization for phase shift and amplitude damping appear unrelated, i.e. one can simplify the of exercise finding $\epsilon$ without mention of $\rho$.

Inset 2: The Rossby equation

The simplest expression for the phase speed of Rossby waves is $\mathbb{C}=\mathrm{U}-\beta / \mathrm{K}^{2}$, where $\mathbb{C}$ is phase speed, $U$ is the background windspeed, $\beta$ is the meridional derivative of the Coriolis parameter, and $\mathrm{K}$ is wavenumber (if only the zonal wavenumber is considered $\mathrm{K}$ relates to $\mathrm{m}$ as follows: $\mathrm{K}=2 \pi \mathrm{m} / \mathrm{L}$, where $\mathrm{L}$ is circumference of the earth.). This was derived by Rossby and collaborators in 1939. A more complete expression is $\mathbb{C}=U-\left(\beta-\partial^{2} U / \partial y^{2}+F^{2} U\right) /\left(K^{2}+F^{2}\right)$, where $K$ is the three dimensional wavenumber, $\mathrm{F}$ is the inverse of the Rossby radius of deformation, and $\beta^{*}=\beta$ $\partial^{2} \mathrm{U} / \partial \mathrm{y}^{2}$ is the apparent $\beta$-effect. Ignoring the vertical wavenumber, and setting the meridional wavenumber 1 equal to $n \pi / \operatorname{acos} \phi$ (and taking $n=3$, independent of $m$ ) the wavespeeds at $50 S$ and $50 \mathrm{~N}$ in Tables 3.1 and 3.2 are actually to within $1.5 \mathrm{~m} / \mathrm{s}$ from theory, i.e. in quantitative agreement with the Rossby equation. Likewise we found the seasonal cycle in phase speed along $50 \mathrm{~N} / \mathrm{S}$ to be well explained by the seasonal cycle in $U$, and $\beta^{*}$.

The energy travels with the group speed $\left(\mathrm{c}_{\mathrm{gx}}, \mathrm{c}_{\mathrm{gy}}\right)$ which can be derived from the above by differentiation wrt wavenumber. We find $c_{\mathrm{gx}}=\mathrm{U}+\left(\beta^{*}+\mathrm{F}^{2} \mathrm{U}\right)\left(\mathrm{k}^{2}-\mathrm{l}^{2}\right) /\left(\mathrm{K}^{2}+\mathrm{F}^{2}\right)^{2}$ and $\mathrm{c}_{\mathrm{gy}}=2 \beta^{*}(\mathrm{kl}) /\left(\mathrm{K}^{2}+\mathrm{F}^{2}\right)^{2}$, see Pedlosky $(1979, \mathrm{p} 114$, chapter 3). Clearly group speed is higher in the zonal than in the meridional direction, see also comments in Chapter 4 about the shape of eddies. As for an expression on the sphere we obtained 


$$
\mathbb{C}=\mathrm{U}_{\text {eq }}-\frac{2\left(\Omega \mathrm{a}+\mathrm{U}_{\text {eq }}\right)+\mathrm{a}^{2} \mathrm{~F}^{2} \mathrm{U}_{\text {eq }}}{\left(\mathrm{n}(\mathrm{n}+1)+\mathrm{a}^{2} \mathrm{~F}^{2}\right)}
$$

where, $\Omega$ is the rate of rotation of the earth, and a is the radius of the earth. This expression holds only for a simple background flow $\mathrm{U}(\phi)=\mathrm{U}_{\mathrm{eq}} \cos \phi$, also called super rotation. The phase speed given is valid at the equator. Interestingly, phase speed depends theoretically on n only. However, in Table 3.5 we established empirically that phase speed depends nearly as much on $\mathrm{m}$.

Ch. 3 Pg. 18 

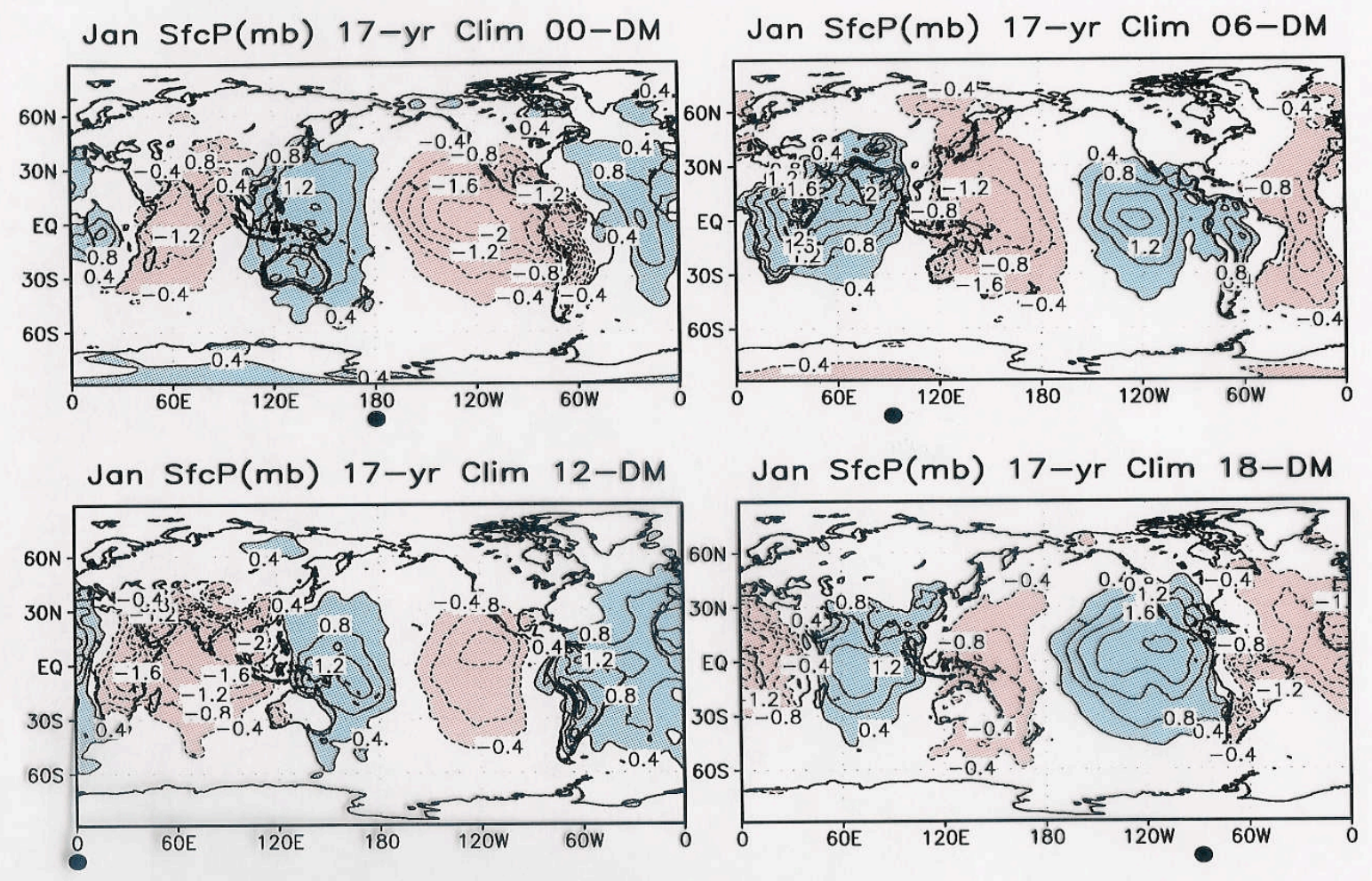

Above: Surface pressure tides in Reanalysis at 0, 6, 12 and 18Z. By making a weighted average of EWP forward and backward, tides can be estimated at any time in between, see next page. Black dot is the position of the sun.

Ch. 3 Pg. 19 


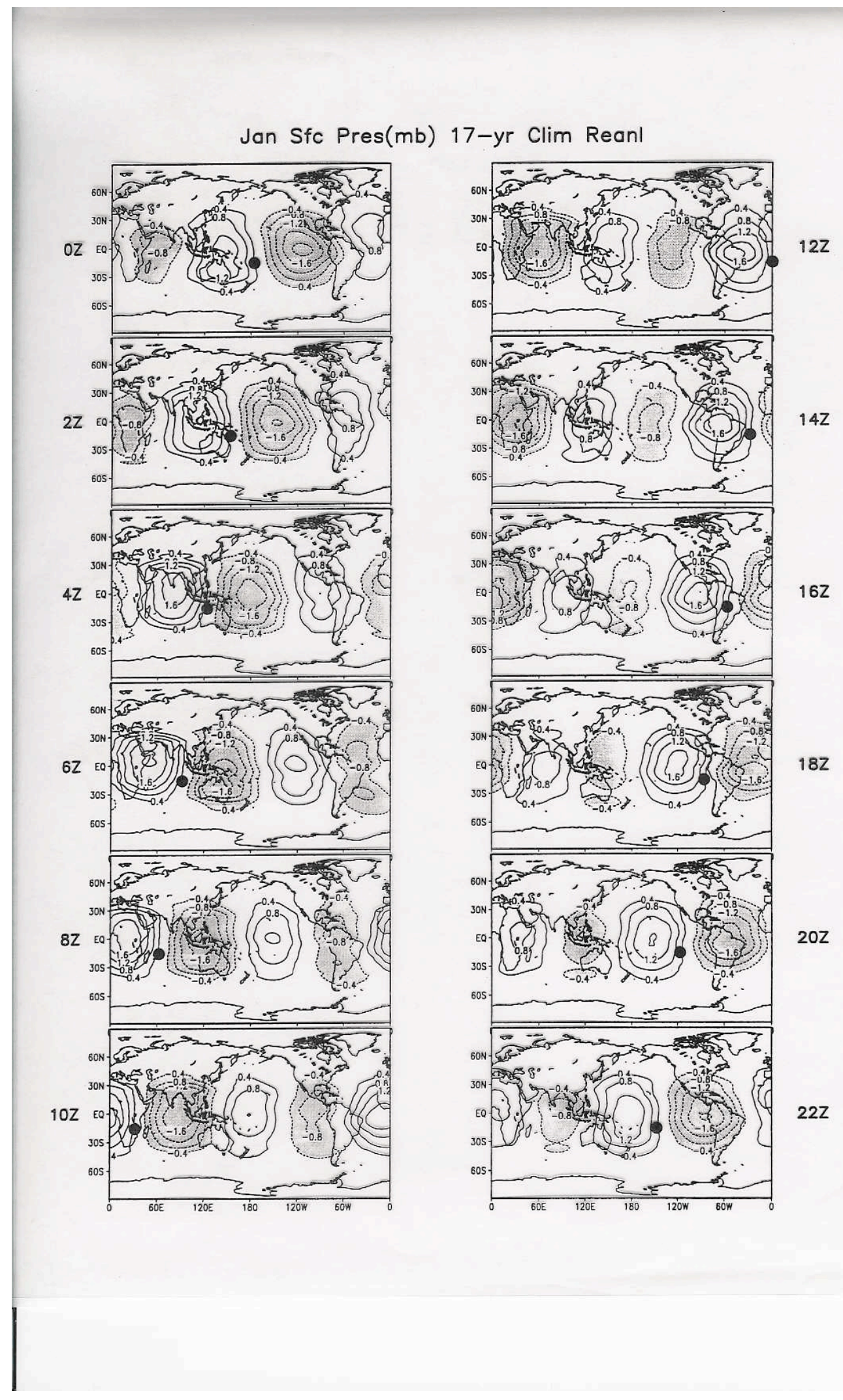

Ch. 3 Pg. 20 